\title{
Dermoscopy of Poikilodermatous Mycosis Fungoides in Pigmented Skin
}

\author{
${ }^{1}$ Ankit H Bharti, ${ }^{2}$ Uday S Khopkar
}

\begin{abstract}
Mycosis fungoides (MF) is one of the most common primary cutaneous T-cell lymphoma, whose early stages, especially patch stage poses a diagnostic challenge, as it presents quite similarly to other inflammatory skin diseases and chronic dermatoses. We present a case of a 31-year-old young male presented to us with complaints of generalized hyperpigmentation associated with mild itching since 4 years. On examination, generalized well-demarcated hyperpigmented poikilodermatous patches with focal atrophy were noted. The poikiloderma was more pronounced over the arms and legs. Dermoscopy of these patches displayed multiple well-demarcated irregular accentuation of pigment network with criss-cross white streaked (loss of pigment network) zones with microscaling, telangiectasia (reddish smudges), and black dots. Histopathology showed interface dermatitis, epidermotropism of atypical lymphocytes, and tagging of lymphocytes along the basement membrane and Pautrier's micro- abscesses. These findings confirmed the diagnosis of poikilodermatous MF. This is the first report of findings of Poikilodermatous MF in pigmented skin. Thus, dermoscopy being a non-invasive tool with specific characteristics, may assume an supplementary role in the diagnosis of poikilodermatous MF.
\end{abstract}

Keywords: Dermoscopy, Mycosis fungoides, Poikilodermatous mycosis fungoides.

How to cite this article: Bharti AH, Khopkar US. Dermoscopy of Poikilodermatous Mycosis Fungoides in Pigmented Skin. Int J Dermoscop 2017;1(1):28-31.

Source of support: Nil

Conflict of interest: None

\section{INTRODUCTION}

Mycosis fungoides (MF) is the most common primary cutaneous T-cell lymphoma (CTCL), a proliferation of memory CD4+ T-cells. The MF in early stages, especially patch stage, poses a diagnostic challenge, as it mimics other inflammatory skin diseases like eczema,

${ }^{1}$ Fellow, ${ }^{2}$ Professor and Head

1,2Department of Dermatology, Seth Gordhandas Sunderdas Medical College and King Edward Memorial Hospital, Mumbai Maharashtra, India

Corresponding Author: Ankit H Bharti, Fellow, Department of Dermatology, Seth Gordhandas Sunderdas Medical College and King Edward Memorial Hospital, Mumbai, Maharashtra, India e-mail: dr.ankithbharti@gmail.com psoriasis or pityriasis lichenoides chronica. ${ }^{1}$ Histopathologic diagnosis of MF has false positivity and false negativity rates of about $40 \% .^{2}$ Poikilodermatous MF is a rare distinct clinical variant of CTCL, formerly referred to as poikiloderma vasculare atrophicans or parapsoriasis variegata. ${ }^{3}$ Herein we report dermoscopic findings in one such case.

\section{CASE REPORT}

A 31-year-old young unmarried male presented to us with complaint of generalized hyperpigmentation with sparing of scalp, face, and hands associated with mild itching since 4 years. The patient was diagnosed as a patch stage MF in 2013 and was given Psoralen + Ultraviolet a therapy from January 2014 to January 2015 for 1 year after which the patient was lost to follow-up and since then he has been off any kind of oral or topical treatment. This was followed by appearance of new lesions, along with increase in pigmentation and size of preexisting patches that spread over to neck, trunk, back, buttocks, bilateral arms, and legs (Figs 1 and 2).

Skin examination showed generalized well-demarcated hyperpigmented poikilodermatous patches with sparing of skin over scalp, hands, and face. The hyperpigmentation over trunk and back was reticulate with focal atrophy (Fig. 3). The poikiloderma was more pronounced over the arms and legs.

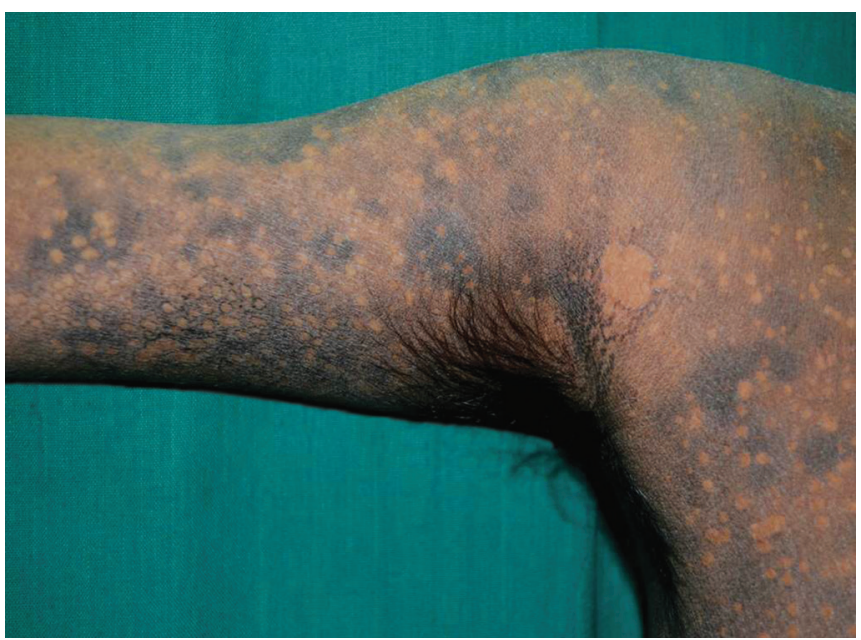

Fig. 1: Right arm and shoulder showing poikilodermatous patches confluent poikilodermatous patched 


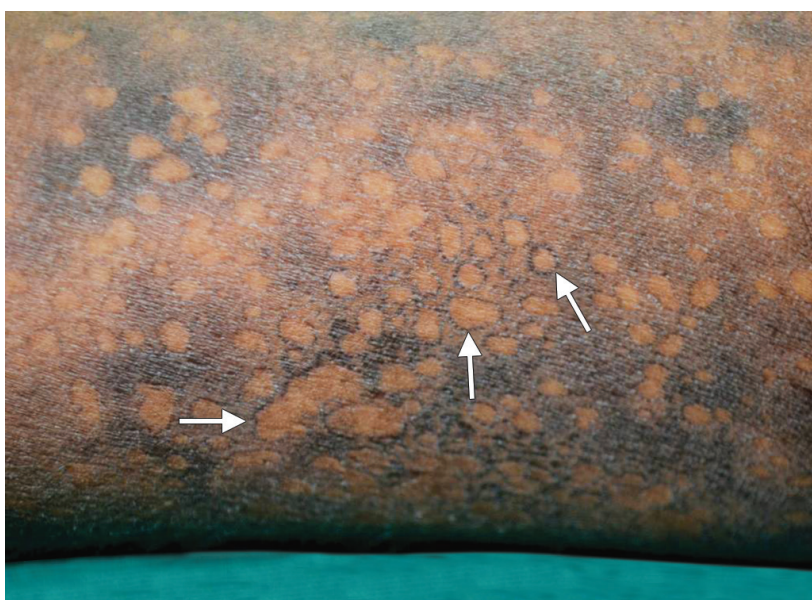

Fig. 2: Right arm showing annular lesions with central atrophy suggestive of poikiloderma

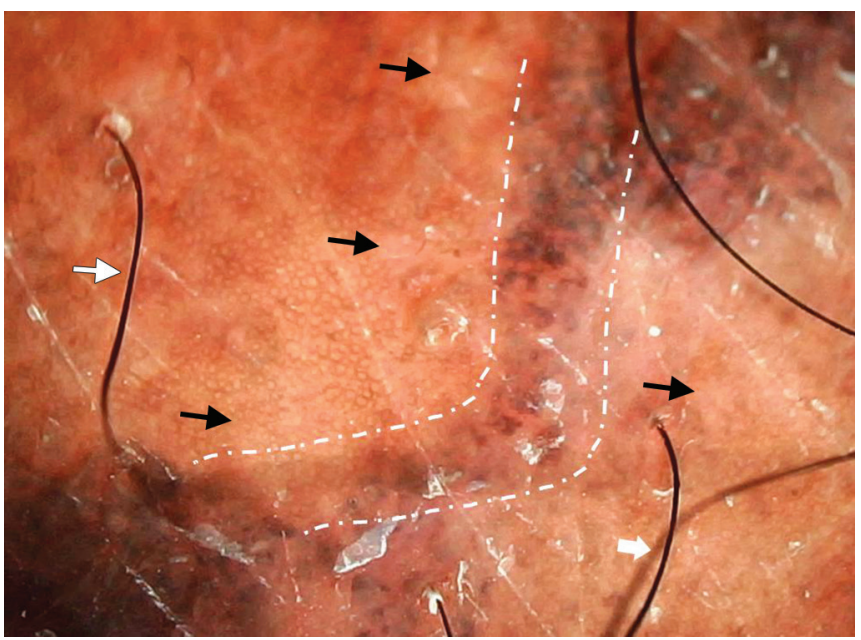

Fig. 4: Dermoscopic image at $(40 \times)$ shows curvilinear streaks of irregularly shaped pigment globules (white dashed line) along with whitish globules (loss of pigment network) obliterating the normal pigment network (black arrow). Red smudges follicles, microscaling, and relative lack of epidermal appendages

\section{Dermoscopic Findings}

Dermoscopy of the hyperpigmented lesions on the legs (at 40x) showed curvilinear streaks of irregularly shaped pigment globules crisscross white streaked (loss of pigment network) zones with microscaling, telangiectasia (reddish smudges), and black dots (Fig. 4).

Higher magnification (200×) revealed well-demarcated irregular accentuation of pigment network along with whitish globules (loss of pigment network) obliterating the normal pigment network (Fig. 5) was observed. It also displayed red smudges(ref 5 in superscript) (telangiectasia - red dotted boxes)along with microscaling and relative lack of epidermal appendages.

Dermoscopy of the annular lesions over arms (40x) displayed a beaded necklace-like appearance (resembling to an extent a lesion of superficial porokeratosis) with inner hypopigmented blotches and atrophy with patchy

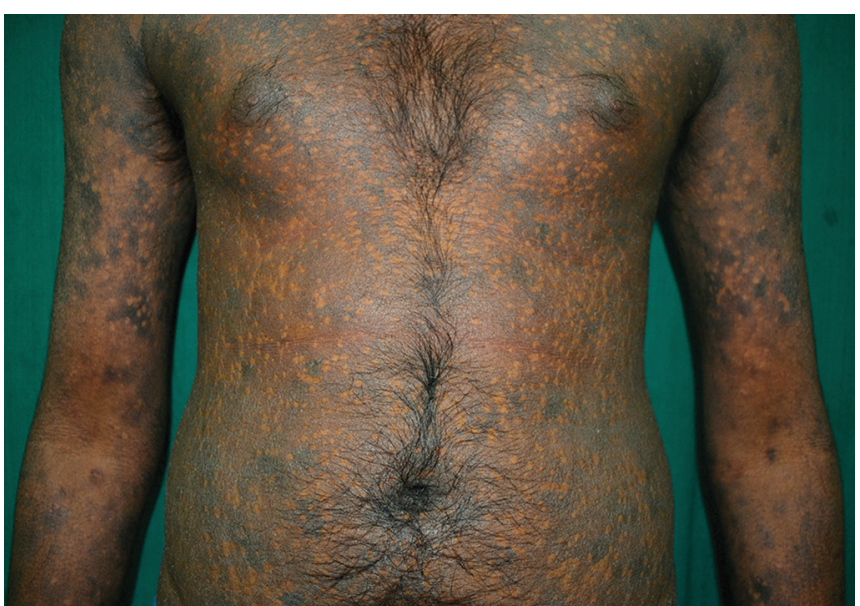

Fig. 3: Hyperpigmented poikilodermatous patches over trunk

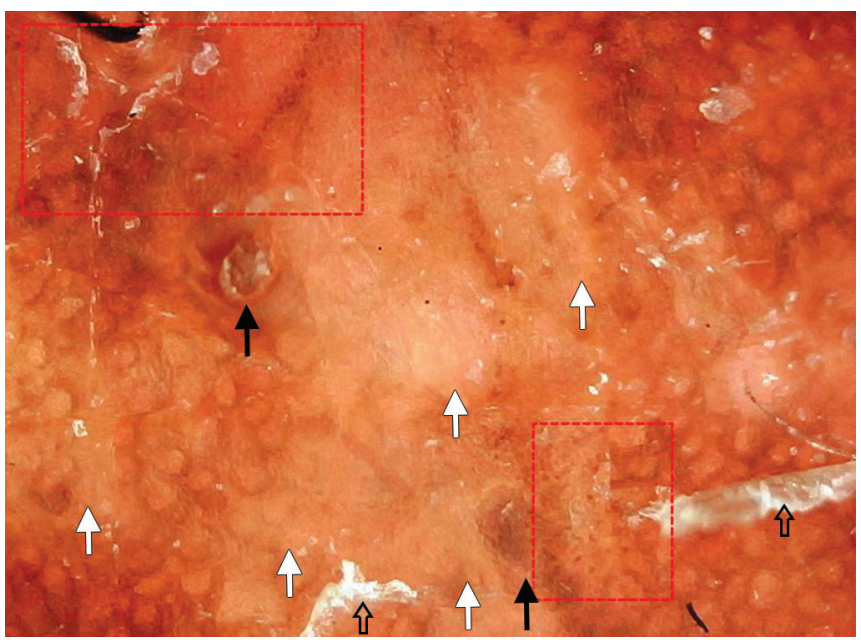

Fig. 5: Dermoscopic image of hyperpigmented reticulate over leg at $(200 \times)$ shows irregular accentuation of pigment network with pigment globules and crisscross white streaked (loss of pigment network) zones with microscaling (black outlined arrows), telangiectasia (reddish smudges — red dotted boxes), and black dots (black solid arrows)

accentuation of pigment network and outer areas that displayed irregular hyperpigmentation with keratin plugs (Fig. 6). The margin of which at (200x) displayed a curvilinear streak with pigment globules (arrows), patchy pigment loss, and atrophy in the center with red dots near margin (Fig. 7).

\section{Histopathological Findings}

Skin biopsy specimen showed mild epidermal hyperplasia with lymphocytic lichenoid interface dermatitis and epidermotropism of atypical lymphocytes with relative lack of spongiosis (Fig. 8). There was vacuolar alteration of the basal layer without evident spongiosis and scattered melanophages in the papillary dermis. There was tagging of atypical large lymphocytes along the basement membrane with a few larger lymphocytes located even above the stratum malphigii rest of the epidermis 


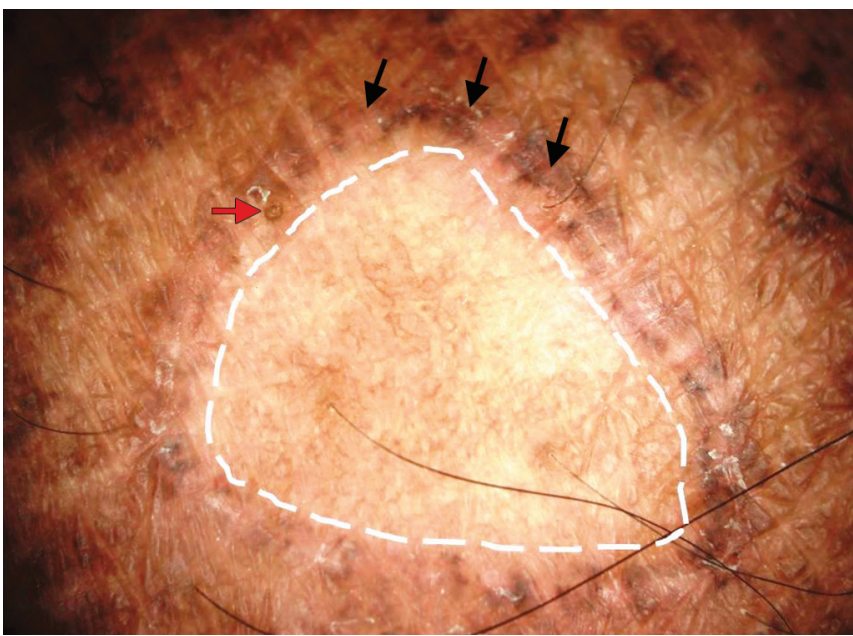

Fig. 6: Dermoscopic image of annular lesion labelled in Fig. 2 displayed a beaded (black arrows) necklace-like appearance (white dashed line) with inner hypopigmented blotches and atrophy with patchy accentuation of pigment network and outer areas display irregular accentuation of pigment network with follicular plugging (red arrow)

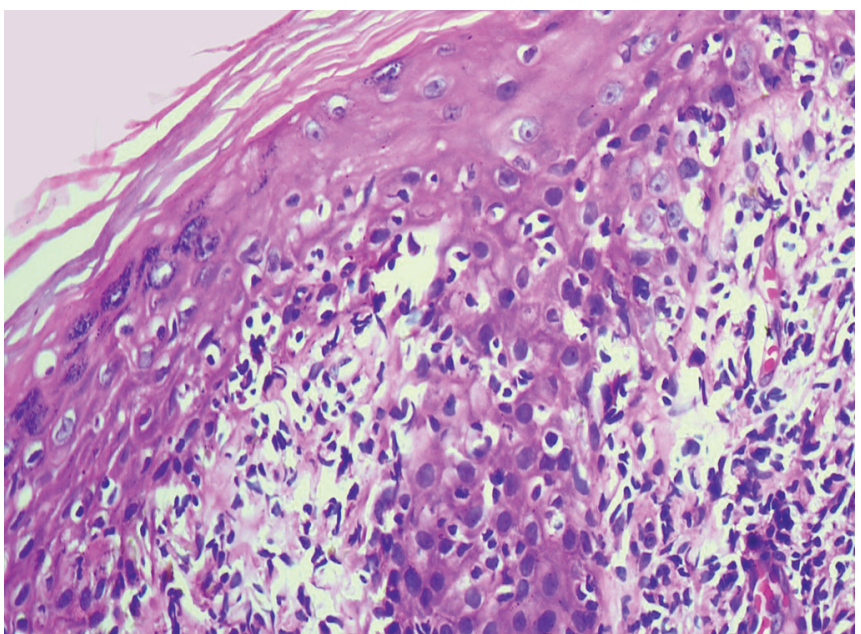

Fig. 8: Histopathology (H\&E 200x) of annular lesion in Fig. 2 showed lichenoid interface dermatitis and epidermotropism of atypical lymphocytes, tagging of atypical large lymphocytes along the basement membrane with several larger lymphocytes located even above the stratum malphigii

was peppered with lymphocytes. There was a clear halo around the lymphocytes with some atypical lymphocytes coalescing to form Pautrier's Pautrier's micro- abscesses (Fig. 9). The size of lymphocytes in epidermis was larger and cerebriform as compared to those in dermis. In view of these findings, a diagnosis of poikilodermatous MF patch stage was made.

\section{DISCUSSION}

Dermoscopy of hypopigmented MF has been described by Lallas et al, wherein an analysis of 67 dermoscopic images was carried out retrospectively. They described presence of dotted vessels, fine short linear vessels, spermatozoa-like structures, orange-yellowish patchy areas,

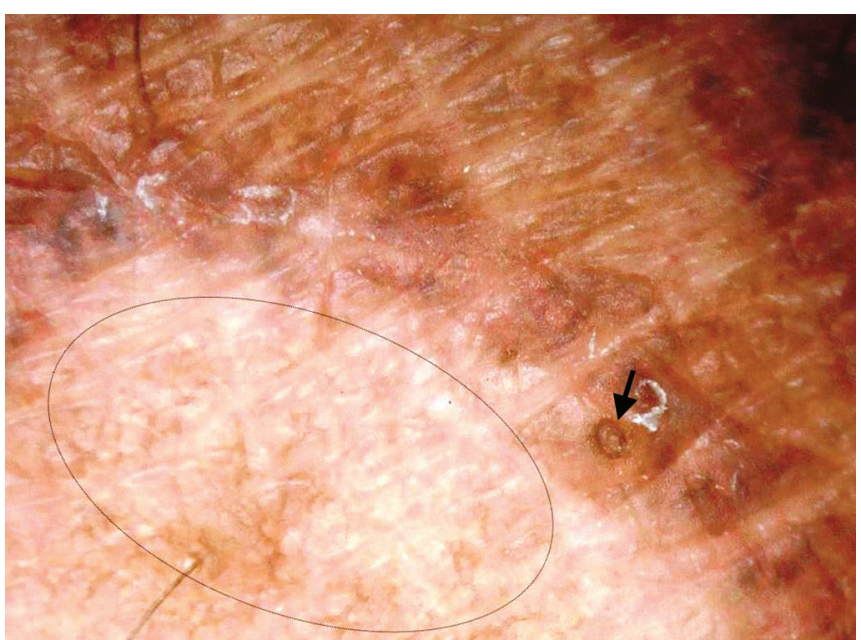

Fig. 7: Dermoscopic image of the margin of annular lesion in Fig. 6 displayed a beaded appearance with pigment globules and inner zone displaying hypopigmented blotches and atrophy (black dotted oval) and outer areas displaying irregular accentuation of pigment network with keratin plug (black arrow)

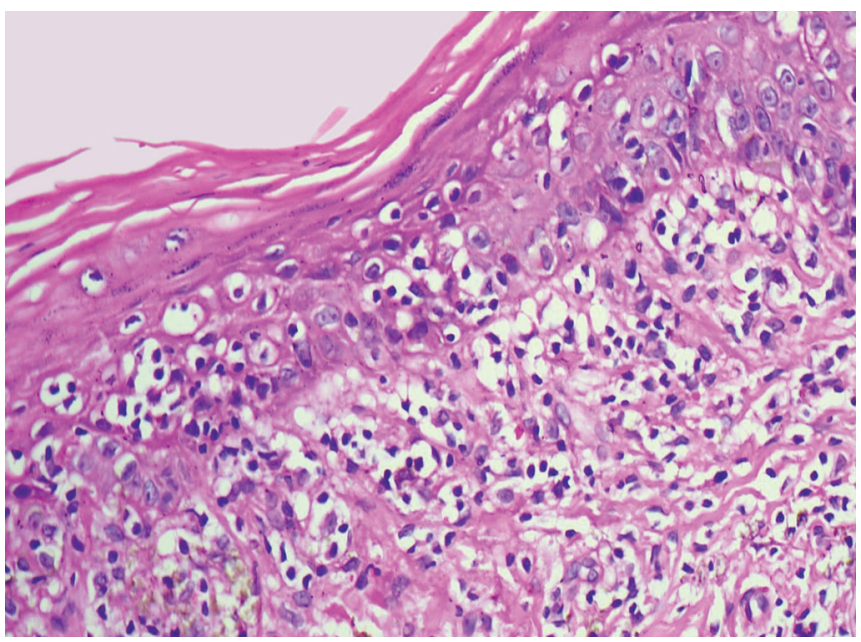

Fig. 9: Histopathology (H\&E 200x) Halos around large lymphoctes and formation of Pautrier's micro- abscesses.

white scales, and yellow scales. ${ }^{1}$ Similarly, in an Egyptian study of dermoscopy of dermatoses, clinically similar to MF were compared with plaque type psoriasis (PP), chronic dermatitis and lichen planus (LP) by Marwah et al. Besides the above findings, they noted that dark globules and light brown multifocal pigmentation on a pink homogeneous background are highly suggestive of MF and that dermoscopy can be an essential tool to differentiate MF from other PP, chronic dermatitis, and LP. ${ }^{4}$

Dermoscopy of pokilodermatous MF has not been documented in Indian skin and the only reference available is in a Chinese patient as described by $\mathrm{Xu}$ et al. They described multiple polygonal structures containing hypopigmented lobules with white storiform streaks, studded with fine red dots as seen in our patient. In addition, their patient also showed hairpin vessels on the 
margins. ${ }^{5}$ The septa between these lobules of pigmented dots showed unevenly and intermittently distributed black dots along with red and yellowish smudges that indicate telagiectasia. Hypochromia in MF was previously associated with well-defined clinical forms, such as poikilodermatous MF or resulting from phototherapy treatment as described by Choe et al. ${ }^{6}$

Considering the clinical dermoscopy and histopathology, the features are complementary in making the diagnosis of poikilodermatous MF. Dermoscopy being a noninvasive tool with specific characteristics, may assume an important role in the diagnosis of poikilodermatous MF. This is the first report of Poikilodermatous MF in Pigmented skin.

\section{REFERENCES}

1. Lallas A, Apalla Z, Lefaki I, Tzellos T, Karatolias A, Sotiriou E, Lazaridou E, Ioannides D, Zalaudek I, Argenziano G. Dermoscopy of early stage mycosis fungoides. J Eur Acad Dermatol Venereol. 2013 May;27(5):617-621.
2. Olsen E, Vonderheid E, Pimpinelli N, Willemze R, Kim Y, Knobler R, Zackheim H, Duvic M, Estrach T, Lamberg S, et al. Revisions to the staging and classification of mycosis fungoides and Sezary syndrome: a proposal of the International Society for Cutaneous Lymphomas (ISCL) and the cutaneous lymphoma task force of the European Organization of Research and Treatment of Cancer (EORTC). Blood 2007 Sep 15;110(6):1713-1722.

3. Pankratov O, Gradova S, Tarasevich S, Pankratov V. Poikilodermatous mycosis fungoides: clinical and histopathological analysis of a case and literature review. Acta Dermatovenerol Alp Pannonica Adriat 2015;24(2):37-41.

4. Saleh MA, Halim DM. Dermoscopy: an easy, noninvasive tool for distinguishing mycosis fungoides from other inflammatory mimics. J Egypt Women's Dermatol Soc 2014 Sep 1;11(3):215-219.

5. Xu P, Tan C. Dermoscopy of poikilodermatous mycosis fungoides (MF). J Am Acad Dermatol 2016 Mar;74(3): e45-e47.

6. Choe YB, Park KC, Cho KH. A case of hypopigmented mycosis fungoides. The Journal of dermatology. 2000 Aug 1;27(8): 543-546. 